\title{
Nuclear Physics and Medicine
}

\section{Modern High-Voltage Equipment in the United States and on the Continent of Europe}

\author{
By L. G. Grimmett, Radium Beam Therapy Research, London*
}

$\mathrm{M}^{\mathrm{s}}$ ANY interesting developments in radiology may be expected during the next few years, as a consequence of the discovery of the neutron and of artificial radioactivity.

The neutron has peculiar and novel properties which may make it of particular value in radiation therapy. To mention only two : it is more heavily absorbed in the hydrogenous tissues of the body than in the bones, and it ionizes by means of recoil protons which give densely ionized tracks, in striking contrast to the characteristics of $\mathrm{X}$ and gamma-radiation, where absorption is roughly proportional to the density of the absorbing medium, and ionization proceeds by means of thinly ionized recoil electron tracks. For these reasons alone it might be anticipated that neutrons would produce different biological effects from $\mathrm{X}$-rays. Indeed, the preliminary experiments of Lawrence and his collaborators on the effects of intense neutron beams on wheat seedlings, mouse tumours and other living organisms, indicate that neutrons are more effective biologically than $\mathrm{X}$-rays, and what is perhaps more significant for therapeutical purposes, that they have a greater 'selective action' than X-rays; that is to say, they inflict relatively greater damage on malignant cells than on normal ones.

Artificial radio-elements have already proved their worth as chemical and biological indicators. Elements can be 'labelled' by mixing with them a small quantity of a radioactive isotope, and their subsequent history traced through the most complicated chemical actions by means of the associated radioactivity. In this way, for example, the metabolism of calcium and phosphorus in the body of the rat have been studied.

Now that it is becoming possible to produce short-lived radioactive isotopes of the common elements in large quantities, measurable in millicuries, we may hope to extend the scope of chemotherapy by the use of radioactive organic compounds, the therapeutic action of which would depend on their radioactivity rather than on their chemical properties.

The recent advances in pure physics are thus of considerable interest to medical science, and I was glad, therefore, to have had the opportunity,

- Published by permission of the Executive Committee of the Radium Beam Therapy Research, London. through the generosity of the Prophit Trust of the Royal College of Surgeons, of visiting many institutions abroad to see what progress had been made in this kind of work. My impressions are given below; it is only possible in this short article to summarize the chief points of interest.

\section{Production of Intense Neutron Beams and OF RADIO-ELEMENTS}

The methods commonly employed for producing intense neutron beams are the bombardment of deuterium, beryllium or lithium by swift deuterons, the probable reactions being :

$$
\begin{aligned}
& \text { 1. }{ }_{1}^{2} \mathrm{D}+{ }_{1}^{2} \mathrm{D} \rightarrow{ }_{2}^{3} \mathrm{He}+{ }_{0}^{1} n \\
& \text { 2. }{ }_{4}^{8} \mathrm{Be}+{ }_{1}^{2} \mathrm{D} \rightarrow{ }_{5}^{10} \mathrm{~B}+{ }_{0}^{1} n \\
& \text { 3. }\left\{\begin{array}{l}
{ }_{3}^{7} \mathrm{Li}+{ }_{1}^{2} \mathrm{D} \rightarrow{ }_{4}^{8} \mathrm{Be}+{ }_{0}^{1} n \\
{ }_{8}^{7} \mathrm{Li}+{ }_{1}^{2} \mathrm{D} \rightarrow{ }_{2}^{4} \mathrm{He}+{ }_{0}^{1} n
\end{array}\right.
\end{aligned}
$$

The efficiency of the last three reactions increases rapidly with the speed of the impinging deuterons.

Neutrons are also liberated in large numbers when beryllium is bombarded with gamma rays of energy greater than 1.4 million volts.

Radio-elements can be synthesized in a great variety of ways, by bombarding elements with neutrons, protons, deuterons or helium nuclei. Here again, the neutron method apart, the eff. ciency of most reactions increases rapidly with the energy of the bombarding particles. It is essential, therefore, to have means of accelerating light nuclei by potentials of the order 1-5 million volts.

The chief types of apparatus for producing high. energy particles are :

1. Cascade transformer. This was one of the earliest types of high-voltage generators, developed by Lauritsen at Pasadena in 1928 for X-ray production at 750 kilovolts, and extended later to 1 million volts. The greatest voltage which has been reached anywhere by this method is about 2 million. The apparatus is large and costly and requires a special building to house it. Currents up to 30 milliamperes can easily be obtained, but they are alternating, which is a disadvantage for many purposes. It is doubtful whether any more cascaded transformers will be built for nuclear physics, in view of the rapid developments of alternative methods. 
2. The cyclotron. The cyclotron of Lawrence accelerates light nuclei to very high speeds by multiple accelerations through a small potential of 20,000 volts or so. Two $D$-shaped electrodes, fed with high-frequency alternating current, are placed in a strong magnetic field which causes the particles to curve back repeatedly through the accelerating field. In this way protons and deuterons can attain speeds corresponding to

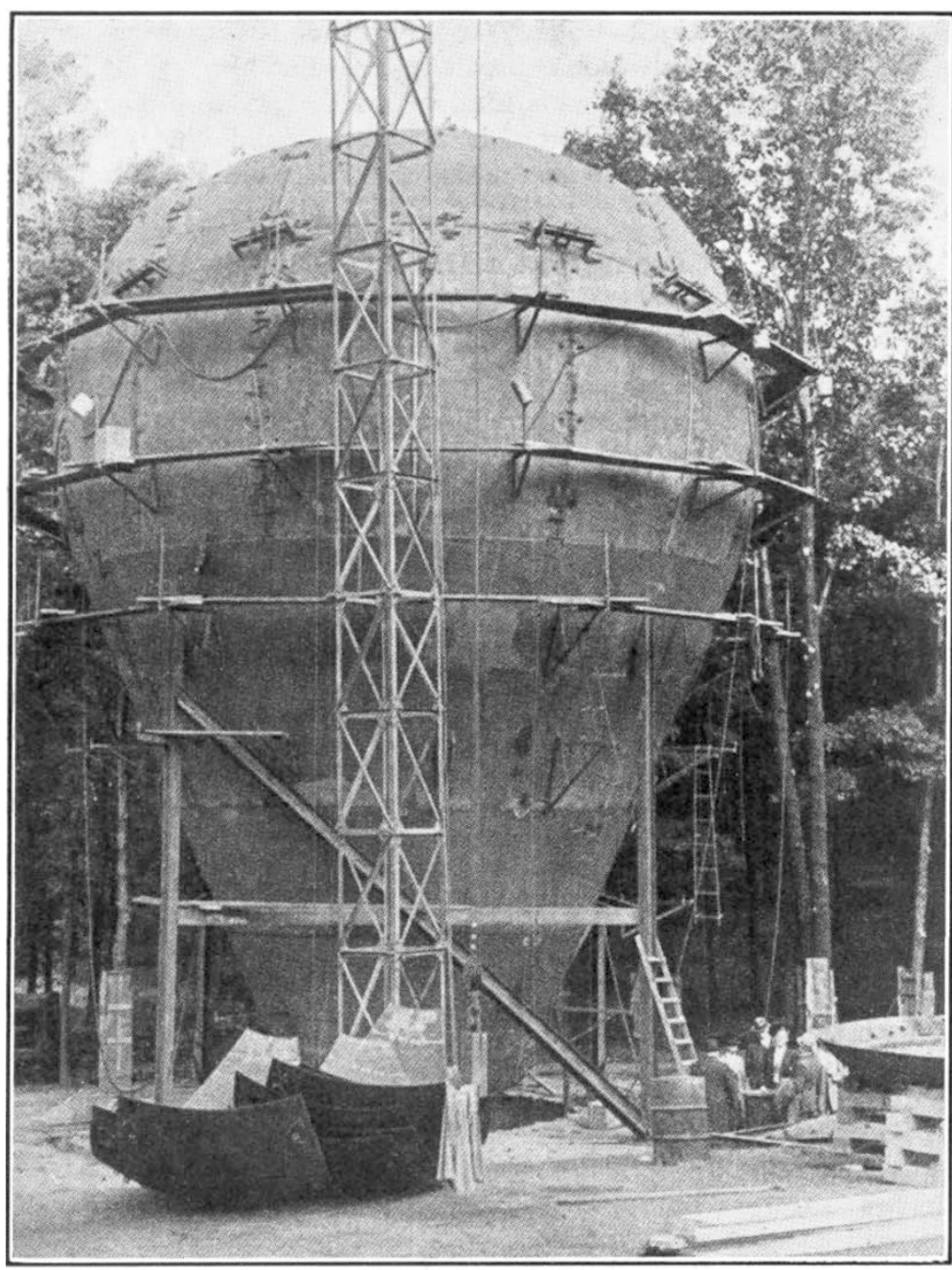

The 5 million volt electrostatic generator of the Carnegie Institution, Washington, D.C., in course of construction (SePtemBER 1937). The charging belts and ion accelerating tube will be mounted vertically inside the pear-shaped tank 55 feet high by $37 \frac{1}{2}$ feet in diameter, and worked under an air pressure of $50 \mathrm{lb} . / \mathrm{sq}$. in. The target and observation room are underground for protection purposes. (By kind permission of the Director.)

5 million volts and more. Using ion currents less than 100 microamperes, the cyclotron has furnished the most powerful neutron beams yet obtained, with which most of the biological work to date has been carried out. Its size is remarkably small for its performance; the whole apparatus, complete with high-frequency oscillator for supplying the electrodes, can be placed in a room of modest dimensions. The power consumption is heavy, about 30 kilowatts being required for the electromagnet and 70 kilowatts for the oscillator. The magnet may weigh as much as 100 tons. The cost is high, and a skilled staff is required to run it.

The cyclotron is not, of course, suitable for accelerating electrons for $\mathrm{X}$-ray production. There are about 25 cyclotrons being built in the world : two of them are in England (Cambridge and Liverpool), intended primarily for physics research.

3. Electrostatic generator. Through the efforts of numerous workers in the United States, the old-fashioned electrostatic generator of the Holtz type has been revived and developed into a formidable high-voltage generator. A well-insulated hollow conductor is charged by swiftly moving endless belts of silk or rubber on which electric charges have been sprayed by corona discharge from a set of points kept at a potential of 10,000 20,000 volts. The voltage attainable is limited only by the breakdown of the surrounding air or by sparking along the insulators, the current being proportional to the size, number and speed of the charging belts. A large generator of this kind has been installed at the Massachusetts Institute of Technology, developing 5 million volts (one terminal 2.5 million volts positive, the other 2.5 million volts negative, to earth) and a current of 2 milliamperes.

A great advance was made by Herb, Parkinson and Kerst, of Madison University, Wisconsin, who enclosed an electrostatic generator together with an ion accelerating tube in an earthed steel tank, and worked them under an air pressure of 100 lb./sq. in. By this means, the breakdown voltage was increased to nearly four times its value at atmospheric pressure and a steady voltage was obtained of $2 \cdot 1$ millions to earth in a tank $20 \mathrm{ft}$. long by $6 \mathrm{ft}$. in diameter. Naturally, in this form the apparatus is independent of the humidity of the external air. 
The design of this and other high-voltage machines is likely to be influenced by the discovery of the remarkable insulating properties of the gas $\mathrm{CCl}_{8} \mathrm{~F}_{2}$, known commercially as a refrigerant under the name "freeon". The breakdown voltage of freeon is two to three times greater than air, and by using this gas under pressure it is possible to increase the breakdown voltage by a factor of 10 .

Ture, of the Carnegie Institution of Washington, D.C., is building a large electrostatic generator working in compressed air (see accompanying illustration). It is expected that voltages in excess of 5 millions will be obtained. The target of the ion tube is at earth potential and is underground, for protection purposes.

The electrostatic generator is relatively cheap, simple and robust. It is beginning to be adopted for hospital use; the Huntingdon Memorial Hospital, Boston, has installed one to work an $\mathrm{X}$-ray tube at 1.2 million volts, 3 milliamperes.

4. Resonance generator. So far this ingenious apparatus, due to Sloan, has only been applied to $X$-ray production. Insulation difficulties are avoided by hanging a copper coil from one end in an evacuated earthed steel tank, about $4 \mathrm{ft}$. across, and making it resonate with a 100 kilowatt radiofrequency oscillator mounted above it, but not connected to it. High-frequency oscillations are induced in the coil, and an alternating potential of about 1 million volts is developed at the free end of the coil, which carries a target. The target faces a hot filament in the wall of the tank, which is biased so that electrons are accelerated only during the peak values of the alternating voltage. The apparatus is compact, but the power consumption is great (100 kilowatts). There is one of these generators in San Francisco, and another at Columbia University, New York.

5. Cascade generators (voltage multipliers). Greinacher, Cockcroft and Walton, and Bouwers, developed independently the cascade generator, an arrangement of condensers and rectifying valves by means of which an alternating potential of 200 kilovolts or so may be multiplied many times and rectified.

Bouwers has succeeded in getting 1.5 million volts constant potential to earth by this method at Philips' Laboratories, Eindhoven, and contemplates 2 million volts to earth in the near future.

The apparatus is on the large side and is expensive, but can be bought, ready for use, and certainly has an impressive performance. It is independent of climatic conditions, has an ample reserve of power, and costs little to run.

At the moment, no ion tube is available at Eindhoven which will stand up to these voltages, but Bouwers, Heyn and Kuntke have constructed ion tubes to work at 300 and 600 kilovolts, yielding more than 1 milliampere of positive ion current, giving neutrons equivalent to $100 \mathrm{gm}$. of radium and beryllium. Even this, however, is a rather small neutron output for biological work, and some of the biological results obtained with it were criticized at the Fifth International Congress of Radiology (Chicago 1937), on these grounds.

Prof. P. Debye is installing a Siemens cascade generator in the new Kaiser Wilhelm Institute at Dahlem, for studying the materialization of gamma rays; it is in two halves, each giving 1.4 million volts to earth, with a total of 2.8 millions between the ends.

6. Impulse generators. Impulse generators working up to 2 million volts have been used for a long time in electrical engineering for the testing of transformers and insulators. The method consists of charging a number of condensers in parallel to 50,000 volts or so, and then discharging them in series, when a momentary voltage equal to the sum of voltages of the separate condensers is obtained. The apparatus is usually quite big and necessitates a special building, the output is under imperfect control, and is not suitable for exact physical experiments. Prof. F. Joliot, however, has turned it to good account in the manufacture of artificial radio-elements. At the Laboratoire Ampère, Paris, he has an impulse generator capable of giving 3 million volts, which is applied to an oil-immersed multi-sectioned $\mathrm{X}$-ray tube of the Brasch and Lange type, about $1 \frac{1}{4}$ metres long. The target of the tube is surrounded by beryllium. The heavy bursts of X-rays which occur at each impulse liberate photo-neutrons from the beryllium, which activate elements placed near by. Quantities of radio-elements approaching a millicurie have been obtained in this manner, and it is hoped to increase the yield by improving the tube design.

Incidentally, Prof. Joliot has solved the difficult problem of selecting the best apparatus for nuclear physics by installing one of each! His new Laboratory of Nuclear Physics, Paris, which is nearing completion, will have at its disposal a cyclotron, cascade generator, 2 million volt cascade transformer, 5 million volt electrostatic generator (recently a popular spectacle at the Palais de la Découverte of the Paris Exposition 1937) together with the impulse generator just mentioned. An extensive scheme of biological research is being planned in collaboration with the Radium Institute of Paris.

High-voltage $X$-ray equipment. X-ray apparatus for therapeutical purposes is still being developed towards higher and higher voltages, in the belief that the shorter wave-lengths are superior in their biological effects. There is a welcome tendency 
towards reducing the dimensions of the equipment. Bouwers described at the Congress of Radiology a million-volt installation, with sealed-off $\mathrm{X}$-ray tube, measuring only $10 \mathrm{ft} . \times 8 \mathrm{ft} . \times 6 \mathrm{ft}$. high.

When the voltage has been pushed a little higher we shall be within sight of an efficient substitute for the natural gamma rays of radium (the radiation spectrum of which extends to about 2 million volts), at a price within the reach of the smaller hospitals, which cannot afford to buy large quantities of radium.

The main impression left by this tour is of the vigour with which the new physical knowledge is being followed up abroad, and applied to biology. One cannot but be struck with the absence of similar efforts in Great Britain, where, apart from a 400 kilovolt neutron generator which is being built at Mount Vernon Hospital, Middlesex, and the 1 million volt X-ray plant at St. Bartholomew's Hospital, London, no work of this kind is going on at all.

It is to be deplored that there is no institution in England where these important physico-medical problems can be attacked with the thoroughness they deserve.

\title{
Resonance and Mesomerism
}

\author{
By Prof. C. K. Ingold, F.R.S.
}

$\mathbf{M}^{\mathrm{s}}$ ESOMERISM is an extension of valency theory, and, like all valency theory, is founded in the quantum theory.

It may be recalled that in the quantum theory it is shown to be impossible accurately to specify the position and motion of an electron at each instant of time. What can be known about an electron, considered as a particle, is of a statistical nature, and is summarized in the behaviour of a wave. The frequency of the wave measures the energy of the electron $(E=h \nu)$ and the amplitude measures the probability that the electron is in a given elemen. tary volume (prob. $=|\psi|^{2} d v$ ). The fundamental wave-property in the theory of the atom is selfinterference-the self-interference of a confined wave ; this annihilates all frequencies except those that can form standing waves, which are perpetuated and describe the quantum states. The fundamental wave-property in the theory of valency is resonance-the resonance of connected standing waves; their mutual perturbation replaces these waves by new standing waves, one of which usually corresponds to the creation or modification of some bond.

In order to appreciate the significance of resonance in the theory of valency, it is useful to recall the nature of resonance in vibrating mechanical systems. The simplest example is that of two like pendulums hung from the same stretched string. If one pendulum is given a displacement, its interaction with the string causes the vibratory motion gradually to become transferred to the second pendulum, after which it travels back to the first (Fig. 1). This motion is obviously not simple harmonic, but it can be shown to be a mixture of two simple harmonic motions. One of these occurs alone if we start the pendulums with equal amplitudes in phase; the frequency $v-\varepsilon$ is then a little less than the frequency $v$ of either uncoupled pendulum, because the two pendulums when swinging in phase deform the supporting string in mutually accommodating ways and this lowers the restoring forces. The other simple harmonic motion occurs when we start the pendulums with equal amplitudes in opposite phase ; the frequency $v+\varepsilon$ is now a little greater than that of the uncoupled pendulums because of the opposing actions on the string. When we start the pendulums with any arbitrary amplitudes and phase difference, the two simple harmonic motions occur together, and the 'beats' arising from their differing frequencies give rise to the complicated motion described.

If $\psi_{1}$ and $\psi_{2}$ are the co-ordinates measuring the displacements of the two pendulums, then before coupling, each co-ordinate was associated with a definite frequency $\nu$, but after coupling neither co-ordinate has any definite frequency. On the other hand, the linear combination $\psi_{1}+\psi_{2}$ has the frequency $\nu-\varepsilon$, whilst the combination $\psi_{1}-\psi_{3}$ has frequency $\nu+\varepsilon$. We may say that the mutual perturbation or 'resonance' has replaced the former co-ordinates of periodic motion $\left(\psi_{1}\right.$ and $\left.\psi_{2}\right)$ by two independent linear combinations $\left(\psi_{1}+\psi_{2}\right.$ and $\left.\psi_{1}-\psi_{2}\right)$, and has split the original coincident frequency $(v)$ into two frequencies, one greater and one less than the original.

In Fig. 1, $(A)$ represents the uncoupled equivalent pendulums. Their displacement co-ordinates are $\psi_{1}$ and $\psi_{2}$, and their motion is periodic, each pendulum having the frequency ${ }^{\circ}(B)$ and $(C)$ 